\title{
Some Operations on Quaternion Numbers
}

\author{
Bo $\mathrm{Li}$ \\ Qingdao University of Science \\ and Technology \\ China \\ Xiquan Liang \\ Qingdao University of Science \\ and Technology \\ China
}

\author{
Pan Wang \\ Qingdao University of Science \\ and Technology \\ China \\ Yanping Zhuang \\ Qingdao University of Science \\ and Technology \\ China
}

Summary. In this article, we give some equality and basic theorems about quaternion numbers, and some special operations.

MML identifier: QUATERN3, version: $7.11 .02 \quad 4.120 .1050$

The articles [11], [1], [12], [3], [4], [9], [2], [5], [8], [7], [10], [13], and [6] provide the notation and terminology for this paper.

In this paper $z_{1}, z_{2}, z_{3}, z_{4}, z$ are quaternion numbers.

The following propositions are true:

(1) $\Re\left(z_{1} \cdot z_{2}\right)=\Re\left(z_{2} \cdot z_{1}\right)$.

(2) If $z$ is a real number, then $z+z_{3}=\Re(z)+\Re\left(z_{3}\right)+\Im_{1}\left(z_{3}\right) \cdot i+\Im_{2}\left(z_{3}\right)$. $j+\Im_{3}\left(z_{3}\right) \cdot k$.

(3) If $z$ is a real number, then $z-z_{3}=\left\langle\Re(z)-\Re\left(z_{3}\right),-\Im_{1}\left(z_{3}\right),-\Im_{2}\left(z_{3}\right)\right.$, $\left.-\Im_{3}\left(z_{3}\right)\right\rangle_{\mathbb{H} \cdot}$.

(4) If $z$ is a real number, then $z \cdot z_{3}=\left\langle\Re(z) \cdot \Re\left(z_{3}\right), \Re(z) \cdot \Im_{1}\left(z_{3}\right), \Re(z) \cdot\right.$ $\left.\Im_{2}\left(z_{3}\right), \Re(z) \cdot \Im_{3}\left(z_{3}\right)\right\rangle_{\mathbb{H}}$.

(5) If $z$ is a real number, then $z \cdot i=\langle 0, \Re(z), 0,0\rangle_{\mathbb{H}}$.

(6) If $z$ is a real number, then $z \cdot j=\langle 0,0, \Re(z), 0\rangle_{\mathbb{H}}$.

(7) If $z$ is a real number, then $z \cdot k=\langle 0,0,0, \Re(z)\rangle_{\mathbb{H}}$.

(8) $z-0_{\mathbb{H}}=z$. 
(9) If $z$ is a real number, then $z \cdot z_{1}=z_{1} \cdot z$.

(10) If $\Im_{1}(z)=0$ and $\Im_{2}(z)=0$ and $\Im_{3}(z)=0$, then $z=\Re(z)$.

(11) $|z|^{2}=(\Re(z))^{2}+\left(\Im_{1}(z)\right)^{2}+\left(\Im_{2}(z)\right)^{2}+\left(\Im_{3}(z)\right)^{2}$.

(12) $|z|^{2}=|z \cdot \bar{z}|$.

(13) $|z|^{2}=\Re(z \cdot \bar{z})$.

(14) $2 \cdot \Re(z)=\Re(z+\bar{z})$.

(15) If $z$ is a real number, then $\overline{z \cdot z_{1}}=\bar{z} \cdot \overline{z_{1}}$.

(16) $\overline{z_{1} \cdot z_{2}}=\overline{z_{2}} \cdot \overline{z_{1}}$.

(17) $\left|z_{1} \cdot z_{2}\right|^{2}=\left|z_{1}\right|^{2} \cdot\left|z_{2}\right|^{2}$.

(18) $i \cdot z_{1}-z_{1} \cdot i=\left\langle 0,0,-2 \cdot \Im_{3}\left(z_{1}\right), 2 \cdot \Im_{2}\left(z_{1}\right)\right\rangle_{\mathbb{H}}$.

(19) $i \cdot z_{1}+z_{1} \cdot i=\left\langle-2 \cdot \Im_{1}\left(z_{1}\right), 2 \cdot \Re\left(z_{1}\right), 0,0\right\rangle_{\mathbb{H}}$.

(20) $j \cdot z_{1}-z_{1} \cdot j=\left\langle 0,2 \cdot \Im_{3}\left(z_{1}\right), 0,-2 \cdot \Im_{1}\left(z_{1}\right)\right\rangle_{\mathbb{H}}$.

(21) $j \cdot z_{1}+z_{1} \cdot j=\left\langle-2 \cdot \Im_{2}\left(z_{1}\right), 0,2 \cdot \Re\left(z_{1}\right), 0\right\rangle_{\mathbb{H}} \cdot$

(22) $k \cdot z_{1}-z_{1} \cdot k=\left\langle 0,-2 \cdot \Im_{2}\left(z_{1}\right), 2 \cdot \Im_{1}\left(z_{1}\right), 0\right\rangle_{\mathbb{H}} \cdot$

(23) $k \cdot z_{1}+z_{1} \cdot k=\left\langle-2 \cdot \Im_{3}\left(z_{1}\right), 0,0,2 \cdot \Re\left(z_{1}\right)\right\rangle_{\mathbb{H}}$.

(24) $\Re\left(\frac{1}{|z|^{2}} \cdot \bar{z}\right)=\frac{1}{|z|^{2}} \cdot \Re(z)$.

(25) $\Im_{1}\left(\frac{1}{|z|^{2}} \cdot \bar{z}\right)=-\frac{1}{|z|^{2}} \cdot \Im_{1}(z)$.

(26) $\Im_{2}\left(\frac{1}{|z|^{2}} \cdot \bar{z}\right)=-\frac{1}{|z|^{2}} \cdot \Im_{2}(z)$.

(27) $\Im_{3}\left(\frac{1}{|z|^{2}} \cdot \bar{z}\right)=-\frac{1}{|z|^{2}} \cdot \Im_{3}(z)$.

(28) $\frac{1}{|z|^{2}} \cdot \bar{z}=\left\langle\frac{1}{|z|^{2}} \cdot \Re(z),-\frac{1}{|z|^{2}} \cdot \Im_{1}(z),-\frac{1}{|z|^{2}} \cdot \Im_{2}(z),-\frac{1}{|z|^{2}} \cdot \Im_{3}(z)\right\rangle_{\mathbb{H}}$.

(29) $z \cdot\left(\frac{1}{|z|^{2}} \cdot \bar{z}\right)=\left\langle\frac{|z|^{2}}{|z|^{2}}, 0,0,0\right\rangle_{\mathbb{H}} \cdot$

(30) $\Re\left(z_{1} \cdot z_{2}\right)=\Re\left(z_{1}\right) \cdot \Re\left(z_{2}\right)-\Im_{1}\left(z_{1}\right) \cdot \Im_{1}\left(z_{2}\right)-\Im_{2}\left(z_{1}\right) \cdot \Im_{2}\left(z_{2}\right)-\Im_{3}\left(z_{1}\right) \cdot \Im_{3}\left(z_{2}\right)$.

(31) $\Im_{1}\left(z_{1} \cdot z_{2}\right)=\left(\Re\left(z_{1}\right) \cdot \Im_{1}\left(z_{2}\right)+\Im_{1}\left(z_{1}\right) \cdot \Re\left(z_{2}\right)+\Im_{2}\left(z_{1}\right) \cdot \Im_{3}\left(z_{2}\right)\right)-\Im_{3}\left(z_{1}\right) \cdot$ $\Im_{2}\left(z_{2}\right)$.

(32) $\Im_{2}\left(z_{1} \cdot z_{2}\right)=\left(\Re\left(z_{1}\right) \cdot \Im_{2}\left(z_{2}\right)+\Im_{2}\left(z_{1}\right) \cdot \Re\left(z_{2}\right)+\Im_{3}\left(z_{1}\right) \cdot \Im_{1}\left(z_{2}\right)\right)-\Im_{1}\left(z_{1}\right) \cdot$ $\Im_{3}\left(z_{2}\right)$.

(33) $\Im_{3}\left(z_{1} \cdot z_{2}\right)=\left(\Re\left(z_{1}\right) \cdot \Im_{3}\left(z_{2}\right)+\Im_{3}\left(z_{1}\right) \cdot \Re\left(z_{2}\right)+\Im_{1}\left(z_{1}\right) \cdot \Im_{2}\left(z_{2}\right)\right)-\Im_{2}\left(z_{1}\right) \cdot$ $\Im_{1}\left(z_{2}\right)$.

(34) $\left|z_{1} \cdot z_{2} \cdot z_{3}\right|^{2}=\left|z_{1}\right|^{2} \cdot\left|z_{2}\right|^{2} \cdot\left|z_{3}\right|^{2}$.

(35) $\Re\left(z_{1} \cdot z_{2} \cdot z_{3}\right)=\Re\left(z_{3} \cdot z_{1} \cdot z_{2}\right)$.

(36) $|z \cdot z|=|\bar{z} \cdot \bar{z}|$.

(37) $|\bar{z} \cdot \bar{z}|=|z|^{2}$.

(38) $\left|z_{1} \cdot z_{2} \cdot z_{3}\right|=\left|z_{1}\right| \cdot\left|z_{2}\right| \cdot\left|z_{3}\right|$.

(39) $\left|z_{1}+z_{2}+z_{3}\right| \leq\left|z_{1}\right|+\left|z_{2}\right|+\left|z_{3}\right|$.

(40) $\left|\left(z_{1}+z_{2}\right)-z_{3}\right| \leq\left|z_{1}\right|+\left|z_{2}\right|+\left|z_{3}\right|$.

(41) $\left|z_{1}-z_{2}-z_{3}\right| \leq\left|z_{1}\right|+\left|z_{2}\right|+\left|z_{3}\right|$. 
(42) $\left|z_{1}\right|-\left|z_{2}\right| \leq \frac{\left|z_{1}+z_{2}\right|+\left|z_{1}-z_{2}\right|}{2}$.

(43) $\left|z_{1}\right|-\left|z_{2}\right| \leq \frac{\left|z_{1}+z_{2}\right|+\left|z_{2}-z_{1}\right|}{2}$.

(44) $\quad|| z_{1}|-| z_{2}|| \leq\left|z_{2}-z_{1}\right|$.

(45) $\quad|| z_{1}|-| z_{2}|| \leq\left|z_{1}\right|+\left|z_{2}\right|$.

(46) $\left|z_{1}\right|-\left|z_{2}\right| \leq\left|z_{1}-z\right|+\left|z-z_{2}\right|$.

(47) If $\left|z_{1}\right|-\left|z_{2}\right| \neq 0$, then $\left(\left|z_{1}\right|^{2}+\left|z_{2}\right|^{2}\right)-2 \cdot\left|z_{1}\right| \cdot\left|z_{2}\right|>0$.

(48) $\left|z_{1}\right|+\left|z_{2}\right| \geq \frac{\left|z_{1}+z_{2}\right|+\left|z_{2}-z_{1}\right|}{2}$.

(49) $\left|z_{1}\right|+\left|z_{2}\right| \geq \frac{\left|z_{1}+z_{2}\right|+\left|z_{1}-z_{2}\right|}{2}$.

(50) $\left(z_{1} \cdot z_{2}\right)^{-1}=z_{2}^{-1} \cdot z_{1}^{-1}$.

(51) $\bar{z}^{-1}=\overline{z^{-1}}$.

(52) $\left(1_{\mathbb{H}}\right)^{-1}=1_{\mathbb{H} \cdot}$.

(53) If $\left|z_{1}\right|=\left|z_{2}\right|$ and $\left|z_{1}\right| \neq 0$ and $z_{1}^{-1}=z_{2}^{-1}$, then $z_{1}=z_{2}$.

(54) $\left(z_{1}-z_{2}\right) \cdot\left(z_{3}+z_{4}\right)=\left(\left(z_{1} \cdot z_{3}-z_{2} \cdot z_{3}\right)+z_{1} \cdot z_{4}\right)-z_{2} \cdot z_{4}$.

(55) $\left(z_{1}+z_{2}\right) \cdot\left(z_{3}+z_{4}\right)=z_{1} \cdot z_{3}+z_{2} \cdot z_{3}+z_{1} \cdot z_{4}+z_{2} \cdot z_{4}$.

(56) $-\left(z_{1}+z_{2}\right)=-z_{1}-z_{2}$.

(57) $-\left(z_{1}-z_{2}\right)=-z_{1}+z_{2}$.

(58) $z-\left(z_{1}+z_{2}\right)=z-z_{1}-z_{2}$.

(59) $z-\left(z_{1}-z_{2}\right)=\left(z-z_{1}\right)+z_{2}$.

(60) $\left(z_{1}+z_{2}\right) \cdot\left(z_{3}-z_{4}\right)=\left(z_{1} \cdot z_{3}+z_{2} \cdot z_{3}\right)-z_{1} \cdot z_{4}-z_{2} \cdot z_{4}$.

(61) $\left(z_{1}-z_{2}\right) \cdot\left(z_{3}-z_{4}\right)=\left(z_{1} \cdot z_{3}-z_{2} \cdot z_{3}-z_{1} \cdot z_{4}\right)+z_{2} \cdot z_{4}$.

(62) $-\left(z_{1}+z_{2}+z_{3}\right)=-z_{1}-z_{2}-z_{3}$.

(63) $-\left(z_{1}-z_{2}-z_{3}\right)=-z_{1}+z_{2}+z_{3}$.

(64) $-\left(\left(z_{1}-z_{2}\right)+z_{3}\right)=\left(-z_{1}+z_{2}\right)-z_{3}$.

(65) $-\left(\left(z_{1}+z_{2}\right)-z_{3}\right)=\left(-z_{1}-z_{2}\right)+z_{3}$.

(66) If $z_{1}+z=z_{2}+z$, then $z_{1}=z_{2}$.

(67) If $z_{1}-z=z_{2}-z$, then $z_{1}=z_{2}$.

(68) $\left(\left(z_{1}+z_{2}\right)-z_{3}\right) \cdot z_{4}=\left(z_{1} \cdot z_{4}+z_{2} \cdot z_{4}\right)-z_{3} \cdot z_{4}$.

(69) $\left(\left(z_{1}-z_{2}\right)+z_{3}\right) \cdot z_{4}=\left(z_{1} \cdot z_{4}-z_{2} \cdot z_{4}\right)+z_{3} \cdot z_{4}$.

(70) $\left(z_{1}-z_{2}-z_{3}\right) \cdot z_{4}=z_{1} \cdot z_{4}-z_{2} \cdot z_{4}-z_{3} \cdot z_{4}$.

(71) $\left(z_{1}+z_{2}+z_{3}\right) \cdot z_{4}=z_{1} \cdot z_{4}+z_{2} \cdot z_{4}+z_{3} \cdot z_{4}$.

(72) $\left(z_{1}-z_{2}\right) \cdot z_{3}=\left(z_{2}-z_{1}\right) \cdot-z_{3}$.

(73) $z_{3} \cdot\left(z_{1}-z_{2}\right)=\left(-z_{3}\right) \cdot\left(z_{2}-z_{1}\right)$.

(74) $\left(z_{1}-z_{2}-z_{3}\right)+z_{4}=\left(z_{4}-z_{3}-z_{2}\right)+z_{1}$.

(75) $\left(z_{1}-z_{2}\right) \cdot\left(z_{3}-z_{4}\right)=\left(z_{2}-z_{1}\right) \cdot\left(z_{4}-z_{3}\right)$.

(76) $z-z_{1}-z_{2}=z-z_{2}-z_{1}$.

(77) $\quad z^{-1}=\left\langle\frac{\Re(z)}{|z|^{2}},-\frac{\Im_{1}(z)}{|z|^{2}},-\frac{\Im_{2}(z)}{|z|^{2}},-\frac{\Im_{3}(z)}{|z|^{2}}\right\rangle_{\mathbb{H}}$. 
(78) $\frac{z_{1}}{z_{2}}=\left\langle\frac{\Re\left(z_{2}\right) \cdot \Re\left(z_{1}\right)+\Im_{1}\left(z_{1}\right) \cdot \Im_{1}\left(z_{2}\right)+\Im_{2}\left(z_{2}\right) \cdot \Im_{2}\left(z_{1}\right)+\Im_{3}\left(z_{2}\right) \cdot \Im_{3}\left(z_{1}\right)}{\left|z_{2}\right|^{2}}\right.$, $\frac{\left(\Re\left(z_{2}\right) \cdot \Im_{1}\left(z_{1}\right)-\Im_{1}\left(z_{2}\right) \cdot \Re\left(z_{1}\right)-\Im_{2}\left(z_{2}\right) \cdot \Im_{3}\left(z_{1}\right)\right)+\Im_{3}\left(z_{2}\right) \cdot \Im_{2}\left(z_{1}\right)}{\left|z_{2}\right|^{2}}$, $\frac{\left(\Re\left(z_{2}\right) \cdot \Im_{2}\left(z_{1}\right)+\Im_{1}\left(z_{2}\right) \cdot \Im_{3}\left(z_{1}\right)\right)-\Im_{2}\left(z_{2}\right) \cdot \Re\left(z_{1}\right)-\Im_{3}\left(z_{2}\right) \cdot \Im_{1}\left(z_{1}\right)}{\left|z_{2}\right|^{2}}$, $\left.\frac{\left(\left(\Re\left(z_{2}\right) \cdot \Im_{3}\left(z_{1}\right)-\Im_{1}\left(z_{2}\right) \cdot \Im_{2}\left(z_{1}\right)\right)+\Im_{2}\left(z_{2}\right) \cdot \Im_{1}\left(z_{1}\right)\right)-\Im_{3}\left(z_{2}\right) \cdot \Re\left(z_{1}\right)}{\left|z_{2}\right|^{2}}\right\rangle_{\mathbb{H} \cdot}$.

(79) $(i)^{-1}=-i$.

(80) $(j)^{-1}=-j$.

(81) $(k)^{-1}=-k$.

Let $z$ be a quaternion number. The functor $z^{2}$ is defined by:

(Def. 1) $z^{2}=z \cdot z$.

Let $z$ be a quaternion number. One can verify that $z^{2}$ is quaternion.

Let $z$ be an element of $\mathbb{H}$. Then $z^{2}$ is an element of $\mathbb{H}$.

One can prove the following four propositions:

(82) $z^{2}=\left\langle(\Re(z))^{2}-\left(\Im_{1}(z)\right)^{2}-\left(\Im_{2}(z)\right)^{2}-\left(\Im_{3}(z)\right)^{2}, 2 \cdot\left(\Re(z) \cdot \Im_{1}(z)\right), 2 \cdot(\Re(z) \cdot\right.$ $\left.\left.\Im_{2}(z)\right), 2 \cdot\left(\Re(z) \cdot \Im_{3}(z)\right)\right\rangle_{\mathbb{H}} \cdot$

(83) $\left(0_{\mathbb{H}}\right)^{2}=0$.

(84) $\quad\left(1_{\mathbb{H}}\right)^{2}=1$.

(85) $z^{2}=(-z)^{2}$.

Let $z$ be a quaternion number. The functor $z^{3}$ is defined as follows:

(Def. 2) $\quad z^{\mathbf{3}}=z \cdot z \cdot z$.

Let $z$ be a quaternion number. Observe that $z^{3}$ is quaternion.

Let $z$ be an element of $\mathbb{H}$. Then $z^{\mathbf{3}}$ is an element of $\mathbb{H}$.

Next we state several propositions:

(86) $\left(0_{\mathbb{H}}\right)^{3}=0$.

(87) $\left(1_{\mathbb{H}}\right)^{3}=1$.

(88) $(i)^{\mathbf{3}}=-i$.

(89) $(j)^{\mathbf{3}}=-j$.

(90) $(k)^{3}=-k$.

(91) $\quad\left(-1_{\mathbb{H}}\right)^{2}=1$.

(92) $\left(-1_{\mathbb{H}}\right)^{3}=-1$.

(93) $z^{\mathbf{3}}=-(-z)^{3}$.

\section{REFERENCES}

[1] Grzegorz Bancerek. The ordinal numbers. Formalized Mathematics, 1(1):91-96, 1990.

[2] Czesław Byliński. The complex numbers. Formalized Mathematics, 1(3):507-513, 1990.

[3] Czesław Byliński. Functions and their basic properties. Formalized Mathematics, 1(1):5565, 1990.

[4] Czesław Byliński. Functions from a set to a set. Formalized Mathematics, 1(1):153-164, 1990.

[5] Czesław Byliński. Some basic properties of sets. Formalized Mathematics, 1(1):47-53, 1990. 
[6] Fuguo Ge. Inner products, group, ring of quaternion numbers. Formalized Mathematics, 16(2):135-139, 2008, doi:10.2478/v10037-008-0019-x.

[7] Krzysztof Hryniewiecki. Basic properties of real numbers. Formalized Mathematics, 1(1):35-40, 1990.

[8] Xiquan Liang and Fuguo Ge. The quaternion numbers. Formalized Mathematics, 14(4):161-169, 2006, doi:10.2478/v10037-006-0020-1.

[9] Andrzej Trybulec. Enumerated sets. Formalized Mathematics, 1(1):25-34, 1990.

[10] Andrzej Trybulec and Czesław Byliński. Some properties of real numbers. Formalized Mathematics, 1(3):445-449, 1990.

[11] Zinaida Trybulec. Properties of subsets. Formalized Mathematics, 1(1):67-71, 1990.

[12] Edmund Woronowicz. Relations and their basic properties. Formalized Mathematics, 1(1):73-83, 1990.

[13] Edmund Woronowicz. Relations defined on sets. Formalized Mathematics, 1(1):181-186, 1990.

Received October 14, 2008 